\title{
RIGIDITÉ DE QUELQUES GROUPES DE GERMES DE DIFFÉOMORPHISMES
}

\author{
FrédÉRIC TOUZET
}

\begin{abstract}
Using the description of non solvable dynamics by Nakai, we give in this paper a new proof of the rigidity properties of some subgroups of $\operatorname{Diff}(\mathbb{C}, 0)$. The $\mathcal{C}^{\infty}$ case is also considered here.
\end{abstract}

\section{Introduction}

Soient Diff $(\mathbb{C}, 0)$ le groupe des germes de difféomorphismes holomorphes de $(\mathbb{C}, 0)$ et $\widehat{\operatorname{Diff}(\mathbb{C}, 0)}$ son complété formel. Rappelons le résultat suivant, dû à $\mathrm{D}$. Cerveau et $\mathrm{R}$. Moussu.

Théorème 1 ([Ce-Mo]). Soient $G_{1}, G_{2}$ deux sous groupes de $\operatorname{Diff}(\mathbb{C}, 0)$ non résolubles et formellement conjugués, i.e. il existe $\widehat{\theta} \in \mathrm{Diff( \mathbb {C } , 0 )}$ tel que $\widehat{\theta} \circ G_{1} \circ \widehat{\theta}^{-1}=G_{2}$; alors $\widehat{\theta}$ converge.

La preuve s'inspire substantiellement d'un résultat de J.-P. Ramis qui établit qu'une série formelle sommable sur deux niveaux est en réalité convergente et ceci requiert un usage assez élaboré de la transformée de Borel-Laplace. Nous proposons ici une autre démonstration en nous bornant aux propriétés élémentaires des fonctions Gevrey et en utilisant le fait (montré par I. Nakai) que les dynamiques de $G_{1}$ et $G_{2}$ contiennent dans leur adhérence les flots réels de champs de vecteurs naturellement associés aux éléments de ces deux groupes. Mentionnons également que Cerveau et Moussu étendent le Théorème 1 dans la situation où $G_{1}$ est résoluble non exceptionnel. Ce cas ne sera pas abordé ici.

Les questions de rigidité topologiques relatives aux dynamiques non résolubles ont été traitées par Shcherbakov $[\mathbf{S h}]$ et la construction des flots de Nakai permet à ce dernier de retrouver ces résultats. Plus précisément, on a la

2000 Mathematics Subject Classification. 37C05.

Mots-clés. Germes de difféomorphismes, classes Gevrey, flots de Nakai, rigidité formelle et topologique, groupes libres. 
Proposition ([Sh], $[\mathbf{N a}])$. Soient $G_{1}$ et $G_{2}$ deux sous groupes non résolubles de Diff $(\mathbb{C}, 0)$ conjugués par un germe d'homéomorphisme $h$ de $(\mathbb{C}, 0)$; alors $h$ est holomorphe ou antiholomorphe.

Sous des hypothèses que nous explicitons ci-dessous, les preuves de Nakai s'adaptent au cas réel.

Soit $\operatorname{Diff}(\mathbb{R}, 0)$ le groupe des germes de difféomorphismes de classe $\mathcal{C}^{\infty}$ de $(\mathbb{R}, 0)$. Nous noterons ^ l'application, qui à un élément $f \operatorname{de} \operatorname{Diff}(\mathbb{R}, 0)$ associe son développement de Taylor en $0, \widehat{f}(x)=\sum_{n=1}^{+\infty} \frac{f^{(n)}(0)}{n !} x^{n}$. Plus généralement, l'image par l'application ` d'une partie $A$ de $\operatorname{Diff}(\mathbb{R}, 0)$ sera notée $\widehat{A}$. Nous montrons alors le

Théorème 2. Soient $G_{1}$ et $G_{2}$ deux sous groupes de $\operatorname{Diff}(\mathbb{R}, 0)$ conjugués par un germe d'homéomorphisme $h$ de $\mathbb{R}, 0$. On suppose de plus que $\widehat{G_{1}}$ (et donc $G_{1}$ ) est non résoluble. Alors h est un difféomorphisme de classe $\mathcal{C}^{\infty}$ en dehors de l'origine. Si de plus l'on suppose que $h$ préserve l'orientation, il existe des entiers positifs $p$, $q$ premiers entre eux et $f \in \operatorname{Diff}(\mathbb{R}, 0)$ préservant l'orientation tel que pour $x \geq 0$, on ait

$$
h(x)=\left(f\left(x^{\frac{1}{p}}\right)\right)^{q} .
$$

La condition de non résolubilité des groupes de difféomorphismes formels associés est nécessaire, comme l'atteste le

Théorème 3. Il existe deux groupes libres à deux générateurs de germes de difféomorphismes d'une variable réelle topologiquement conjugués mais non conjugués par un germe d'homéomorphisme de $\mathbb{R}, 0$ de classe $\mathcal{C}^{\infty}$ en dehors de l'origine.

Ce travail s'organise de la façon suivante:

Les sections I et II exposent des résultats déjà bien connus sur les fonctions Gevrey et la classification des difféomorphismes (travaux d'Écalle, Martinet, Ramis, Malgrange ... ). A ce titre, le lecteur pourra consulter $[\mathbf{E}],[\mathbf{R a}],[\mathbf{M a}]$. Dans ce travail, nous nous réfèrerons principalement à $[\mathbf{L o}]$ et $[\mathbf{R a}]$.

Les sections III, IV et V sont respectivement consacrées aux preuves des Théorèmes 1,2 et 3.

\section{Développements asymptotiques, classes des fonctions Gevrey}

Soit $f$ une fonction holomorphe dans un ouvert sectoriel

$$
V=\{z \in \mathbb{C}, 0<|z|<r, \arg (z) \in U\}
$$


où $U$ est un ouvert de $S^{1}$. Soit $\sum a_{n} z^{n} \in \mathbb{C}[[z]]$ une série formelle; par définition, on dira que $f$ admet $\sum a_{n} z^{n}$ comme développement asymptotique si pour tout $n$,

$$
f(z)-\sum_{p=0}^{n} a_{p} z^{p}=z^{n} \varepsilon(z) \text { avec } \lim _{\substack{z \in V \\ z \rightarrow 0}} \varepsilon(z)=0 .
$$

Dans ces conditions, on utilisera la notation $f(z) \sim \sum a_{n} z^{n}$.

Si de plus il existe des constantes positives $C, M$ et un entier positif $k$ tels que

$$
\left|f(z)-\sum_{p=0}^{n} a_{p} z^{p}\right| \leq C(n+1) !^{\frac{1}{k}}(M|z|)^{n+1},
$$

on dira que $f$ appartient à la classe $G_{k}(V)$ des fonctions Gevrey d'ordre $k$ sur $V^{*}$. Enfin, on notera $\mathcal{A}^{\leq-k}(V)$ l'ensemble $\left\{f \in G_{k}(V) \mid f \sim 0\right\}$. Remarquons que $G_{k}(V)$ est un sous espace vectoriel de l'espace des fonctions holomorphes sur $V$ et contient la restriction à $V$ des fonctions holomorphes sur le disque $\{|z|<r\}$. Plus généralement, on dira qu'une fonction holomorphe sur un ouvert $U$ de $\mathbb{C}$ est Gevrey d'ordre $k$ sur $U$ $\left(f \in G_{k}(U)\right)$ s'il existe un ouvert sectoriel $V \supset U$ tel que $f$ se prolonge analytiquement en un élément de $G_{k}(V)$.

Proposition 1 (cf. [Ra]). Soit $f \in \mathcal{A}^{\leq-k}(V)$; alors $f$ est une fonction à décroissance exponentielle d'ordre $k$ sur $V$; i.e. il existe des constantes positives $C, M$ telles que

$$
\forall z \in V, \quad|f(z)| \leq C e^{\frac{-M}{|z|^{k}}} .
$$

Réciproquement, toute fonction à décroissance exponentielle d'ordre $k$ sur $V$ est un élément de $\mathcal{A}^{\leq-k}(V)$.

Proposition 2 (cf. [Ra] (Principe de Phragmen-Lindelöf)). Soit $f \in$ $\mathcal{A}^{\leq-k}(V)$, où $V$ est un ouvert sectoriel connexe de la forme $0<|z|<r$, $\theta_{0}<\arg (z)<\theta_{1}, \theta_{1}-\theta_{0}>\frac{\pi}{k}$; alors $f=0$.

Soit $\left(I_{i}\right)_{i \in I}$ un recouvrement fini du cercle $S^{1}$ par des intervalles ouverts choisi de telle sorte que l'intersection de trois $I_{i}$ soit toujours vide. Pour tout $i$, notons $V_{i, r}$ l'ouvert sectoriel $\left\{0<|z|<r, \arg (z) \in I_{i}\right\}$.

Proposition 3 (cf. [Ra]). Supposons, pour tout $i, j \in I$, qu'il existe $\varphi_{i j} \in \mathcal{A}^{\leq-k}\left(V_{i, r} \cap V_{j, r}\right)$ vérifiant $\varphi_{i j}=-\varphi_{j i}$. Soit $r^{\prime}<r$. Alors, pour tout $i \in I$, il existe $\varphi_{i} \in G_{k}\left(V_{i, r^{\prime}}\right)$ tel que $\varphi_{i j}=\varphi_{i}-\varphi_{j}$.

*Dans la définition usuelle, ces estimations s'appliquent sur tout sous secteur relativement compact de $V$ mais ceci est sans importance dans le cas présent. 
Corollaire 4. Soit $\left(\psi_{i}\right)_{i \in I}$ une famille de fonctions holomorphes et bornées sur $V_{i, r^{\prime}}$ telle que $\forall i, j \in I$, on ait

$$
\psi_{i}-\psi_{j}=\varphi_{i j}, \quad \varphi_{i j} \in \mathcal{A}^{\leq-k}\left(V_{i, r^{\prime}} \cap V_{j, r^{\prime}}\right) .
$$

Alors, $\psi_{i} \in G_{k}\left(V_{i, r^{\prime}}\right)$.

Preuve: Il suffit de constater que les $\varphi_{i}-\psi_{i}$ se recollent en une fonction holomorphe sur le disque épointé $0<\left\{|z|<r^{\prime}\right\}$ et s'étendent à l'origine puisque celle-ci devient une singularité artificielle.

\section{Classification formelle et conjugaison sectorielle des éléments de $\operatorname{Diff}(\mathbb{C}, 0)$}

Un calcul formel donne immédiatement le

Lemme 5. Soit $f \in \widehat{\operatorname{Diff}(\mathbb{C}, 0)}$ tangent à l'identité (i.e., $f^{\prime}(0)=1$ ); alors $f$ est l'exponentielle au temps 1 d'un unique champ de vecteurs formel $X_{f}=F(z) \frac{\partial}{\partial z}$ où $F$ est une série formelle d'une variable telle que $F(0)=F^{\prime}(0)=0$, i.e.

$$
f(z)=\sum_{n=0}^{+\infty} \frac{X_{f}^{(n)}}{n !}(z)
$$

où $X_{f}^{(n)}$ désigne le $n^{\text {ieme }}$ itéré de $X_{f}$ vu comme dérivation.

De plus, si on suppose $f$ différent de l'identité, $g \in \mathrm{Diff( \mathbb {C } , 0 )}$ tangent à l'identité commute avec $f$ si et seulement si il existe $t \in \mathbb{C}$ tel que $g=\exp \left(t X_{f}\right)$.

Il est à noter, et c'est d'ailleurs la situation générique, que le champ $X_{f}$ peut diverger en dépit du fait que $f$ converge.

Proposition 6 (Forme normale formelle). Soit $f \in \operatorname{Diff}(\mathbb{C}, 0)$ de la forme $f(z)=z+z^{p+1}+o\left(z^{p+1}\right), p \geq 1$. Alors, il existe $\widehat{\varphi} \in \widehat{\operatorname{Diff}(\mathbb{C}, 0)}$ tangent à l'identite tel que $\widehat{\varphi} \circ f \circ \widehat{\varphi}^{-1}=f_{p, \lambda}$ où $f_{p, \lambda} \in \operatorname{Diff}(\mathbb{C}, 0)$ est le flot au temps 1 du champ de vecteurs $X_{p, \lambda}=\frac{z^{p+1}}{1+\lambda z^{p}} \frac{\partial}{\partial z}$.

La démonstration de ce résultat est basée sur une utilisation simple du théorème des fonctions implicites formel. Elle est similaire au cas $\mathcal{C}^{\infty}$ réel que nous détaillerons dans la partie IV. 
Proposition 7 (Théorème de la fleur de Leau) (cf. [Lo]). Soit $f(z)=$ $z+z^{p+1}+o\left(z^{p+1}\right), p \geq 1$ un germe de $\operatorname{Diff}(\mathbb{C}, 0)$ tangent à l'identité. Soient $\alpha^{\prime}<\alpha$ deux réels de $\left.I_{p}=\right] \frac{\pi}{2 p}, \frac{\pi}{p}\left[\right.$. Pour $x \in I_{p}$ et $r>0$, considérons les domaines sectoriels

$$
V_{k, x, r}=\left\{0<|z|<r, \frac{k \pi}{p}-x<\arg (z)<\frac{k \pi}{p}+x\right\}, \quad k \in \frac{\mathbb{Z}}{2 p \mathbb{Z}} .
$$

Alors, pour $r>0$, il existe $0<r^{\prime}<r$ et des ouverts connexes $\mathcal{P}_{k}, V_{k, \alpha^{\prime}, r^{\prime}} \subset$ $\mathcal{P}_{k} \subset V_{k, \alpha, r}$ tels que $\forall k \in \frac{\mathbb{Z}}{2 p \mathbb{Z}}, \forall z \in \mathcal{P}_{k}, \forall n \geq 0$,

$$
f^{-n}(z) \in \mathcal{P}_{k}, \quad \lim _{n \rightarrow+\infty} f^{-n}(z)=0
$$

si $k$ est pair;

$$
f^{n}(z) \in \mathcal{P}_{k}, \quad \lim _{n \rightarrow+\infty} f^{n}(z)=0
$$

si $k$ est impair.

Remarques. Cet énonce et ceux qui suivent doivent se comprendre pour $r$ "assez petit".

On représente usuellement plutôt les $\mathcal{P}_{k}$ par des ouverts en forme de pétales (dits répulsifs ou attractifs selon que $k$ est pair ou impair) d'où le nom de "Théorème de la fleur" (voir figure ci-dessous avec $p=1,2$ ).
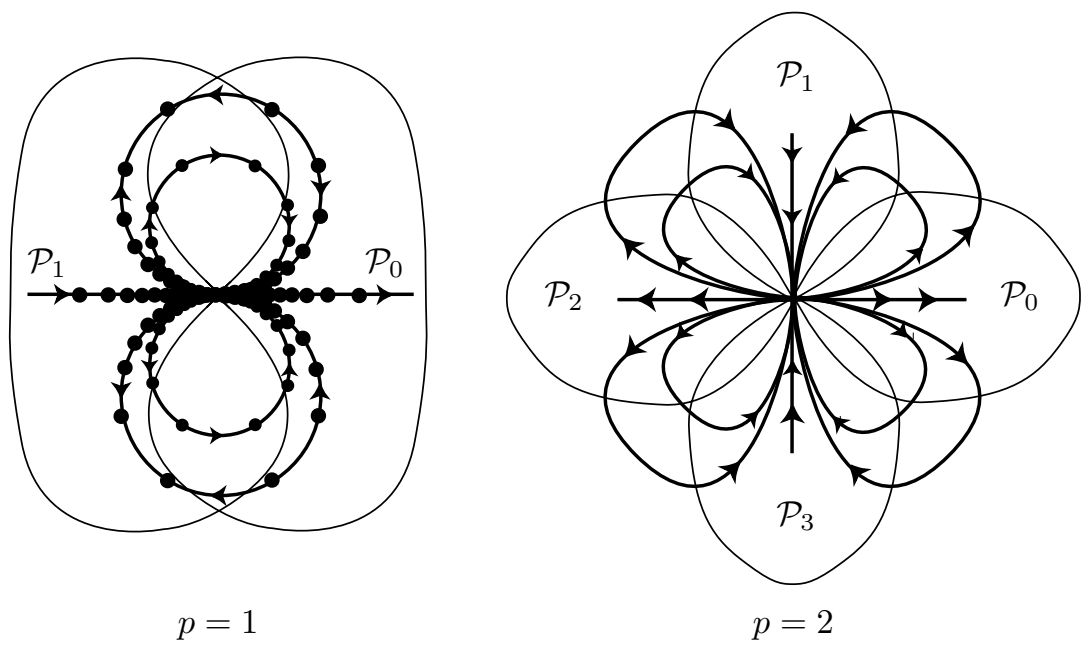

On reprend les notations de la Proposition 7. 
Proposition 8 (Conjugaison sectorielle) (cf. $[\mathbf{M a}],[\mathbf{K}])$. Soient $f_{l}(z)=$ $z+z^{p+1}+o\left(z^{p+1}\right), l=1,2$ deux éléments de $\operatorname{Diff}(\mathbb{C}, 0)$. On suppose qu'il existe un difféomorphisme formel $\widehat{\theta}$ tangent à l'identité conjugant $f_{1} \grave{a} f_{2}$, c'est à dire $f_{2}=\widehat{\theta} \circ f_{1} \circ \widehat{\theta}^{-1}$. Alors, sur chaque pétale $\mathcal{P}_{2 i+1}$ attaché à $f_{1}$, $f_{2}{ }^{-n} \circ f_{1}{ }^{n}$ converge lorsque $n \rightarrow+\infty$, vers un difféomorphisme holomorphe $\varphi_{2 i+1}$ conjugant $f_{1} \grave{a} f_{2}$. On hérite de difféomorphismes $\varphi_{2 i}$ analogues sur $\mathcal{P}_{2 i}$ en considérant la limite de la famille ${f_{2}}^{n} \circ f_{1}{ }^{-n}$. De plus, chaque $\varphi_{k}$ admet sur un ouvert sectoriel contenant $\mathcal{P}_{k}$ un développement asymptotique $\widehat{\varphi}$ tangent à l'identité et indépendant de $k$ conjugant $f_{1}$ $\grave{a} f_{2}$.

Remarque. En appliquant le Lemme 5, on obtient en particulier que $\widehat{\varphi}=\exp \left(t X_{f_{1}}\right) \circ \widehat{\theta}$ pour $t \in \mathbb{C}$.

Considérons maintenant la collection de pétales $\left(\mathcal{P}_{i}^{\prime}\right)=\left(\varphi_{i}\left(\mathcal{P}_{i}\right)\right)$ associée à $f_{2}$ et soit la fonction multivaluée $\psi_{p, \lambda}=-\frac{1}{p z^{p}}+\lambda \log z$.

Supposons d'abord que $f_{2}=f_{p, \lambda}$; on vérifie alors que

— Toute détermination de $\psi_{p, \lambda}$ est univalente en restriction à chaque $\mathcal{P}_{i}{ }^{\prime}$.

- Pour tout $w \in \psi_{p, \lambda}\left(\mathcal{P}_{i}\right), \psi_{p, \lambda_{*}} X_{p, \lambda}=\frac{\partial}{\partial w}$ et en particulier $\psi_{p, \lambda} \circ$ $f_{2} \circ \psi_{p, \lambda}{ }^{-1}(w)=w+1$.

L'ouvert $\psi_{p, \lambda}\left(\mathcal{P}_{i}{ }^{\prime}\right)$ a l'aspect représenté ci-dessous et contient en particulier un demi plan de la forme $\operatorname{Re} w>a$ ou $\operatorname{Re} w<-a$, pour $a \in \mathbb{R}$ positif assez grand, selon que $\mathcal{P}_{i}{ }^{\prime}$ est attractif ou répulsif.

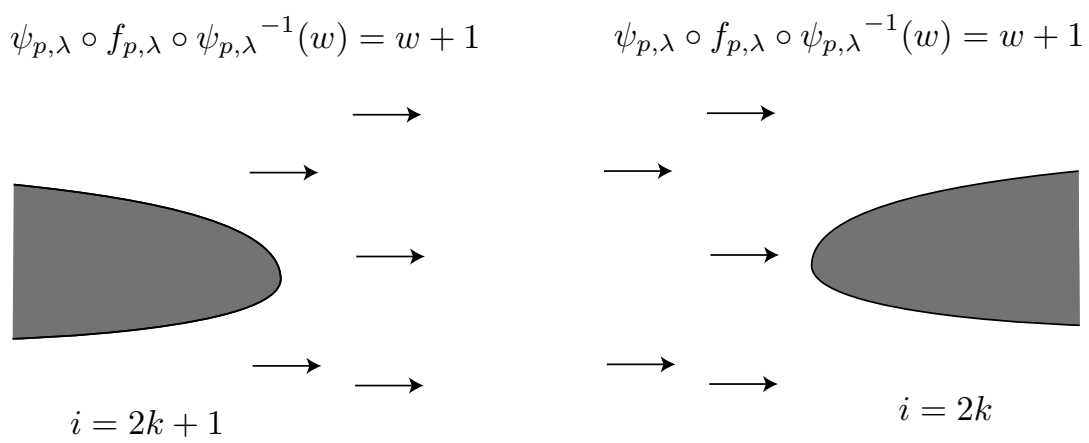

Proposition 9. Sous l'hypothèse $f_{2}=f_{p, \lambda}$ et pour tout $i \in \frac{\mathbb{Z}}{p \mathbb{Z}}$, on a $\varphi_{i} \in G_{p}\left(\mathcal{P}_{i}\right)$. 
Preuve: D'après le Corollaire 4, il suffit de montrer que $\varphi_{i+1}-\varphi_{i} \in$ $\mathcal{A}^{-p}\left(\mathcal{P}_{i} \cap \mathcal{P}_{i+1}\right)$. On pourra supposer $\mathcal{P}_{i}$ répulsif, la démonstration qui suit étant formellement la même dans le cas attractif. Soit $\psi_{i}:=\psi_{p, \lambda} \circ \varphi_{i}$ (défini sur $\mathcal{P}_{i}$ ). On observe alors que $\psi_{i} \circ \psi_{i+1}{ }^{-1}$ est défini sur un domaine du type $\mathcal{D}=\{\alpha<\operatorname{Re} w \leq \alpha+1, \operatorname{Im} w>\beta\}$. Il se prolonge en fait à l'ouvert $\mathcal{D}+\mathbb{N}$ suivant la relation de conjugaison

$$
\psi_{i} \circ \psi_{i+1}^{-1}(w)+1=\psi_{i} \circ \psi_{i+1}^{-1}(w+1) .
$$

Par suite, $\xi_{i}(w+1)=\xi_{i}(w)$ où $\xi_{i}(w)=\psi_{i} \circ \psi_{i+1}{ }^{-1}(w)-w$. Puisque le quotient $\frac{\mathcal{D}+\mathbb{N}}{w \rightarrow w+1}$ est paramétré par $H(w)=e^{2 i \pi w}$, on a $\xi_{i}=\varphi(H)$ où $\varphi$ est holomorphe sur $H\left(\frac{\mathcal{D}+\mathbb{N}}{w \rightarrow w+1}\right)=\left\{u \in \mathbb{C}, 0<|u|<e^{-2 \pi \beta}\right\}$. Par ailleurs, un calcul élémentaire nous donne que $\lim _{\operatorname{Im} w \rightarrow+\infty} \xi_{i}(w)=$ 0 , ce qui entraîne que $\varphi$ est en fait holomorphe en 0 et $\varphi(0)=0$. On obtient donc que $\psi_{p, \lambda} \circ \varphi_{i} \circ \varphi_{i+1}{ }^{-1}=\psi_{p, \lambda}+\varphi\left(e^{2 i \pi \psi_{p, \lambda}}\right) \operatorname{sur} \psi_{p, \lambda}{ }^{-1}(\mathcal{D}+\mathbb{N})$. Posons $\varphi_{i} \circ \varphi_{i+1}{ }^{-1}(z)=z(1+\delta(z))$ où $\delta$ est une fonction plate sur $\varphi_{i+1}\left(\mathcal{P}_{i} \cap \mathcal{P}_{i+1}\right)$. On vérifie alors que sur $\mathcal{P}_{i} \cap \mathcal{P}_{i+1}$ (ou plus exactement sa composante connexe considérée), on a:

$$
\delta(z)=\frac{\varphi\left(z^{\lambda} e^{-\frac{2 i \pi}{z^{P}}}\right)}{\frac{1}{z^{p}}+o(1)} .
$$

On conclut en composant à droite chaque terme de (E) par $\varphi_{i+1}$.

Si $f_{2}$ n'est conjugué que formellement à $f_{p, \lambda}$, on considére la collection de difféomorphismes $\left(\beta_{i}\right)$ définis dans la Proposition 8 conjugant sur chaque pétale $\mathcal{P}_{i}{ }^{\prime} f_{2}$ à $f_{p, \lambda}$. Le difféomorphisme $\psi_{p, \lambda} \circ \beta_{i}$ (défini sur $\mathcal{P}_{i}$ ) est appelé coordonnée de Leau associée à $f_{2}$. Le fait que $\beta_{i}$ admette un développement asymptotique entraîne le

Lemme 10. Pour tout $t \in \mathbb{C}$, il existe un sous-pétale $\mathcal{P}_{i, t}{ }^{\prime}$ de $\mathcal{P}_{i}{ }^{\prime}$ tel que $\exp \left(t X_{i}\right)$, où $X_{i}=\left(\psi_{p, \lambda} \circ \beta_{i}\right)^{*} \frac{\partial}{\partial w}$, soit défini sur $\mathcal{P}_{i, t}{ }^{\prime}$ et admette $\exp \left(t X_{f_{2}}\right)$ comme développement asymptotique.

Corollaire 11. Chaque difféomorphisme $\varphi_{i}$ conjugant $f_{1}$ à $f_{2}$ appartient à $G_{p}\left(\mathcal{P}_{i}\right)$.

Preuve: Il suffit encore de montrer que $\varphi_{i+1}-\varphi_{i} \in \mathcal{A}^{-p}\left(\mathcal{P}_{i} \cap \mathcal{P}_{i+1}\right)$. Soient $\psi_{p, \lambda} \circ \beta_{i}, \psi_{p, \lambda} \circ \alpha_{i}$ les coordonnées de Leau associées respectivement à $f_{2}$ et $f_{1}$ sur $\mathcal{P}_{i}{ }^{\prime}$ et $\mathcal{P}_{i}$. On a $\varphi_{i}=\beta_{i}{ }^{-1} \circ \alpha_{i}$; par suite $\varphi_{i+1}-\varphi_{i}=A_{i}+B_{i}$ avec $A_{i}=\beta_{i+1}{ }^{-1} \circ \alpha_{i+1}-\beta_{i+1}{ }^{-1} \circ \alpha_{i}$ et $B_{i}=\beta_{i+1}{ }^{-1} \circ \alpha_{i}-\beta_{i}{ }^{-1} \circ \alpha_{i}$. La Proposition 9 a établi que $\alpha_{i}$ et $\beta_{i}$ appartiennent respectivement à 
$G_{p}\left(\mathcal{P}_{i}\right)$ et $G_{p}\left(\mathcal{P}_{i}{ }^{\prime}\right)$; par l'inégalite de la moyenne, on a donc que $A_{i} \in$ $\mathcal{A}^{-p}\left(\mathcal{P}_{i} \cap \mathcal{P}_{i+1}\right)$. Par ailleurs, en utilisant que

$$
\beta_{i} \circ \beta_{i+1}{ }^{-1}(z)=z+\gamma(z), \quad \gamma \in \mathcal{A}^{-p}\left(\beta_{i+1} \circ \beta_{i}{ }^{-1}\left(\mathcal{P}_{i} \cap \mathcal{P}_{i+1}\right)\right),
$$

on obtient que $\beta_{i+1}^{-1}(z)-\beta_{i}^{-1}(z)=\beta_{i}^{-1}(z+\gamma(z))-\beta_{i}^{-1}(z)$. En réappliquant l'inégalité de la moyenne au second membre, on obtient que $B_{i} \in$ $\mathcal{A}^{-p}\left(\mathcal{P}_{i} \cap \mathcal{P}_{i+1}\right)$.

Corollaire 12. Pour tout $i$, il existe sur $\mathcal{P}_{i}$ un difféomorphisme $\theta_{i} \in$ $G_{p}\left(\mathcal{P}_{i}\right)$ tel que

$$
\theta_{i} \circ f_{1} \circ \theta_{i}^{-1}=f_{2}, \quad \theta_{i} \sim \widehat{\theta} .
$$

Preuve: Soit $\widehat{\beta} \in \operatorname{Diff(\mathbb {C},0)}$ tel que $\beta_{i} \sim \widehat{\beta}$ (cf. preuve du Corollaire 11). D'après la Proposition 8 , il existe $t \in \mathbb{C}$ tel que $\widehat{\theta}=\widehat{\beta}^{-1} \circ \exp \left(t X_{p, \lambda}\right) \circ \widehat{\beta} \circ \widehat{\varphi}$. On constate alors que $\theta_{i}:=\beta_{i}^{-1} \circ \exp \left(t X_{p, \lambda}\right) \circ \beta_{i} \circ \varphi_{i}$ conjugue sur $\mathcal{P}_{i}$ $f_{1}$ à $f_{2}$. De même que dans la preuve du Corollaire 11, on montre alors que $\theta_{i} \in G_{p}\left(\mathcal{P}_{i}\right)$.

Soient $g_{j} \in \operatorname{Diff}(\mathbb{C}, 0), j=1,2$ de la forme $g_{j}(z)=z+a_{j} z^{q+1}+$ $o\left(z^{q+1}\right), a_{j} \neq 0, q>p$. En réintroduisant les notations du Corollaire 12, considérons la fonction $H_{i}=\theta_{i} \circ g_{1}-g_{2} \circ \theta_{i}$. Puisque les $g_{i}$ sont tangents à l'identité, on peut supposer que $H_{i}$ est défini sur $\mathcal{P}_{i}$.

Proposition 13. Supposons que $\widehat{\theta} \circ g_{1} \circ \widehat{\theta}^{-1}=g_{2}$. Alors, pour tout $i \in \frac{\mathbb{Z}}{2 p \mathbb{Z}}$, on $a H_{i}=0$.

Afin d'établir cette proposition, il sera commode d'exploiter le résultat suivant dont la preuve est analogue à celle du Corollaire 11:

Lemme 14. Pour tout $i \in \frac{\mathbb{Z}}{2 p \mathbb{Z}}$, on a

(i) $\theta_{i} \circ g_{1}-\theta_{i+1} \circ g_{1} \in \mathcal{A}^{\leq-p}\left(\mathcal{P}_{i} \cap \mathcal{P}_{i+1}\right)$.

(ii) $g_{2} \circ \theta_{i}-g_{2} \circ \theta_{i+1} \in \mathcal{A}^{\leq-p}\left(\mathcal{P}_{i} \cap \mathcal{P}_{i+1}\right)$.

Preuve de la Proposition 13: Pour tout $i, \mathcal{P}_{i}$ contient un secteur d'ouverture strictement supérieure à $\frac{\pi}{p}$ et $\widehat{H_{i}}=0$ par hypothèse. Il suffit donc, d'après la Proposition 2, d'établir que $H_{i} \in G_{p}\left(\mathcal{P}_{i}\right)$ et ceci résulte du Lemme 14. 


\section{Preuve du Théorème 1}

Rappelons le fait basique suivant:

Lemme 15. Soit $\widehat{G} \in \mathrm{Diff( \mathbb {C } , 0 )}$ un sous groupe non résoluble. Alors il existe dans $\widehat{G}$ deux éléments, $\widehat{f}$ et $\widehat{g}$, tangents à l'identité à des ordres différents.

$C^{\prime}$ 'est à dire $\widehat{f}(z)-z=a_{p+1} z^{p+1}+o\left(z^{p+1}\right), \widehat{g}(z)-z=b_{q+1} z^{q+1}+o\left(z^{q+1}\right)$, $1 \leq p<q, a_{p+1}, b_{q+1} \neq 0$.

Preuve: Le groupe des commutateurs $[\widehat{G}, \widehat{G}]$ est par hypothèse non abélien; choisissons $\widehat{f}, \widehat{h} \in[\widehat{G}, \widehat{G}]$ non commutants entre eux. On constate alors que $\widehat{f}$ et $\widehat{g}=\widehat{f} \circ \widehat{h} \circ \widehat{f}^{-1} \circ \widehat{h}^{-1}$ satisfont l'énoncé du lemme.

Soit $f \in \operatorname{Diff}(\mathbb{C}, 0)$ satisfaisant les hypothèses du Lemme 10 dont on reprend les notations. Soit $g \in \operatorname{Diff}(\mathbb{C}, 0), g(z)=z+a z^{q+1}+o\left(z^{q+1}\right)$ avec $q>p, a \neq 0$. Rappelons le résultat fondamental suivant, dû à I. Nakai.

Proposition $16([\mathrm{Na}]$, voir aussi $[$ Lo $])$. Posons $k_{n}=n^{\frac{q}{p}-1}$. Pour $x \in \mathbb{R}$, on notera $[x]$ la partie entière de $x$. Soit $K$ un compact du pétale $\mathcal{P}_{2 i+1}$. Alors il existe $T>0$ et $n_{0} \in \mathbb{N}$ tel que, pour tout entier $n \geq n_{0}$, la suite $F_{n}(t, z):=f^{-n} \circ g^{\left[t k_{n}\right]} \circ f^{n}(z)$ soit bien définie et converge uniformément sur $[-T, T] \times K$ vers $\exp t\left(\nu X_{2 i+1}\right)$ où $\nu$ est un nombre complexe.

Cette propriété persiste sur un pétale répulsif $\mathcal{P}_{2 i}$ en considérant la suite $f^{n} \circ g^{\left[t k_{n}\right]} \circ f^{-n}$.

Remarque. Nous redémontrerons partiellement la Proposition 16 dans le cas $\mathcal{C}^{\infty}$ (partie IV).

Soient $G_{i}, i=1,2$ deux sous groupes de $\operatorname{Diff}(\mathbb{C}, 0)$ satisfaisant les hypothèses du Théorème 1 . On peut, quitte à conjuguer ces groupes par des transformations linéaires, supposer que $\widehat{\theta}$ est tangent à l'identité et que $G_{1}$ contient un élément $f_{1}$ s'écrivant sous la forme $f_{1}(z)=z+z^{p+1}+$ $o\left(z^{p+1}\right)$. Compte tenu du Lemme 15, il existe alors dans chaque $G_{i}$ deux difféomorphismes

$$
\begin{aligned}
& f_{i}(z)=z+z^{p+1}+o\left(z^{p+1}\right), \\
& g_{i}(z)=z+a z^{q+1}+o\left(z^{q+1}\right), \quad 1 \leq p<q, a \neq 0,
\end{aligned}
$$

tels que $\widehat{\theta} \circ f_{1} \circ \widehat{\theta}^{-1}=f_{2}$ et $\widehat{\theta} \circ g_{1} \circ \widehat{\theta}^{-1}=g_{2}$. Sur chaque pétale $\mathcal{P}_{i}$ d'un recouvrement associé à $f_{1}$, on peut incarner $\widehat{\theta}$ par un biholomorphisme $\theta_{i}$ conjugant $f_{1}$ à $f_{2}$ et $g_{1}$ à $g_{2}$ (Corollaire 12, Proposition 13). Les couples $\left(f_{1}, g_{1}\right)$ et $\left(f_{2}, g_{2}\right)$ satisfont alors les hypothèses de la Proposition 16 
(les deux collections de pétales considérées étant ici respectivement $\left(\mathcal{P}_{i}\right)$ et $\left(\theta_{i}\left(\mathcal{P}_{i}\right)\right)$. On suppose dorénavant que $i$ est pair. La démonstration $\mathrm{du}$ Théorème 1 résulte alors des points suivants:

a) Le difféomorphisme $\eta_{i}=\theta_{i+1}^{-1} \circ \theta_{i}$ (défini sur $\theta_{i}^{-1} \circ \theta_{i+1}\left(\mathcal{P}_{i} \cap\right.$ $\left.\mathcal{P}_{i+1}\right)$ ) commute à $f_{1}$ et $g_{1}$.

b) Dans la coordonnée de Leau $\psi_{i}=\psi_{p, \lambda} \circ \alpha_{i}$ attachée à $f_{1}$, le difféomorphisme $\tilde{\psi}_{i}=\psi_{i} \circ \eta_{i} \circ \psi_{i}^{-1}$ est défini pour tout $\alpha$ assez grand sur un domaine du type $\mathcal{D}_{\alpha}=\{\alpha<\operatorname{Re} w \leq \alpha+1, \operatorname{Im} w>$ $\left.\beta_{\alpha}\right\}$. Il se prolonge analytiquement en une application univalente sur $\mathcal{D}_{\alpha}+\mathbb{N}$ et a pour expression $\tilde{\psi}_{i}(w)=w+\xi_{i}(w)$ avec $\xi_{i}(w+1)=\xi_{i}(w)$ et $\lim _{\operatorname{Im} w \rightarrow+\infty} \xi_{i}(w)=0$ (cf. preuve de la Proposition 9).

c) Posons $G_{i}:=\psi_{i} \circ g_{1} \circ \psi_{i}^{-1}$. On vérifie que $G_{i}(w)=w+O\left(|w|^{1-\frac{q}{p}}\right)$. Considérons un domaine $\mathcal{D}_{\alpha_{0}}$ défini en b). Il existe alors un domaine du type $\mathcal{D}_{\alpha_{1}} \subset \mathcal{D}_{\alpha_{0}}, \alpha_{1}>\alpha_{0}$ tel que $G_{i}\left(\mathcal{D}_{\alpha_{1}}\right) \subset \mathcal{D}_{\alpha_{0}}$.

d) Pour tout $w \in \tilde{\psi}_{i}\left(\mathcal{D}_{\alpha_{1}}\right), \tilde{\psi}_{i} \circ G_{i} \circ \tilde{\psi}_{i}{ }^{-1}=G_{i}$.

e) Posons $\tilde{F}_{n}(t, w)=G_{i}{ }^{\left[t k_{n}\right]}(w+n)-n$. D'après la Proposition 16 , pour tout compact $K_{1}$ de $\mathcal{D}_{\alpha_{1}}$, il existe un compact $K_{2} \subset \mathcal{D}_{\alpha_{1}}$ tel que $\tilde{F}_{n}\left(t, K_{1}\right) \in K_{2},|t| \leq T>0$ assez petit et $n \geq n_{0}$. En particulier, $G_{i}{ }^{\left[t k_{n}\right]}(w+n) \in \overline{\mathcal{D}}_{\alpha_{1}}$ pour tout $w \in K_{1}$.

f) Des observations qui précèdent, il résulte que pour $t \in[-T, T]$ et $n \geq n_{0}, \tilde{\psi}_{i} \circ F_{n}\left(t, \tilde{\psi}_{i}^{-1}(w)\right)=F_{n}(t, w)$ sur $K_{1}$. Par passage à la limite, on déduit que $\tilde{\psi}_{i *} \frac{\partial}{\partial w}=\frac{\partial}{\partial w}$ pour tout $w \in \mathcal{D}_{\alpha_{0}}$. Finalement, on peut conclure que $\tilde{\psi}_{i}$ est un flot à un certain temps complexe du champ $\frac{\partial}{\partial w}$.

g) Les mêmes remarques valent pour $i$ impair. Sachant que $\eta_{i}$ est plat à l'identité à l'origine, on conclut par le Lemme 10 que les $\theta_{i}$ se recollent en un élément de $\operatorname{Diff}(\mathbb{C}, 0)$.

\section{Preuve du Théorème 2}

Rappelons les résultats suivants, dûs à F. Takens.

Proposition 17 ([Ta]). Soit $X$ un germe de champ de vecteurs de $\mathbb{R}, 0$ de classe $\mathcal{C}^{\infty}$ s'écrivant sous la forme $X=x^{p+1} F(x) \frac{\partial}{\partial x}, p \geq 1, F(0) \neq$ 0. On notera encore

$$
X_{p, \lambda}=\frac{x^{p+1}}{1+\lambda x^{p}} \frac{\partial}{\partial x} .
$$


Alors il existe $\varphi \in \operatorname{Diff}(\mathbb{R}, 0)$ et $(p, \lambda) \in \mathbb{N}^{*} \times \mathbb{R}$ tel que

$$
\begin{aligned}
\varphi_{*} X & =X_{p, \lambda} \quad \text { si } F(0)>0 \\
& =-X_{p, \lambda} \quad \text { si } F(0)<0 .
\end{aligned}
$$

Preuve: Elle s'inspire, comme nous l'avons déjà indiqué, du cas formel et diffère de celle donnée par Takens (dont les formes normales ont d'ailleurs une autre expression). Il suffit évidemment de traiter seulement le cas $F(0)>0$.

Soit $\omega$ la 1-forme duale de $X$. i.e.: $\omega(X)=1$. Elle peut s'écrire

$$
\omega=\left(\frac{1}{F(0) x^{p+1}}+\sum_{i=1}^{p} \frac{a_{i}}{x^{p+1-i}}+O(1)\right) d x
$$

où $a_{i} \in \mathbb{R}$ et le terme $O(1)$ est de classe $\mathcal{C}^{\infty}$. Le difféomorphisme $\varphi$ cherché doit donc vérifier

$$
\frac{1+\lambda \varphi^{p}}{\varphi^{p+1}} d \varphi=\omega
$$

Si on pose $\varphi(x)=x \xi(x)$ et on choisit $\lambda=a_{p}$, on est conduit, via intégration d'une équation différentielle, à résoudre

$$
-\frac{1}{\xi^{p}(x)}+p \lambda x^{p} \ln \xi=p x^{p} \int\left(\sum_{i=1}^{p-1} \frac{a_{i}}{x^{p+1-i}}+O(1)\right) d x-\frac{1}{F(0)} .
$$

Le théorème des fonctions implicites garantit alors l'existence d'une solution $\xi \mathcal{C}^{\infty}$ au voisinage de l'origine telle que $\xi(0)=F(0)^{\frac{1}{p}}$.

Ce principe de mise sous forme normale du champ $X$ permet à Takens d'établir la

Proposition 18 ([Ta]). Soit $f \in \operatorname{Diff}(\mathbb{R}, 0)$ tel que $f(x)=x+a x^{p+1}+$ $o\left(x^{p+1}\right), p \geq 1, a \neq 0$. Alors, il existe un unique champ de vecteurs $X_{f}$ sous la forme donnée par la Proposition 17 dont $f$ est le flot au temps 1.

Plaçons nous sous les hypothèses du Théorème 2. On peut se ramener au cas où $h$ préserve l'orientation. Par ailleurs, $h$ envoie commutateurs sur commutateurs. On peut alors déduire du Lemme 15 l'existence dans chaque groupe $G_{i}$ de deux éléments $f_{i}, g_{i}$ tels que

$$
\begin{aligned}
f_{1}(x) & =x+x^{p_{1}+1}+o\left(x^{p_{1}+1}\right), & g_{1}(x) & =x+a x^{q_{1}+1}+o\left(x^{q_{1}+1}\right), \\
f_{2}(x) & :=h \circ f_{1} \circ h^{-1}(x) & g_{2}(x) & :=h \circ g_{1} \circ h^{-1}(x) \\
& =x+b x^{p_{2}+1}+o\left(x^{p_{2}+1}\right), & & =x+c x^{q_{2}+1}+o\left(x^{q_{2}+1}\right)
\end{aligned}
$$


avec $a<0,0<p_{1}<q_{1}, b>0, c<0$. Quitte à faire les deux substitutions $f_{1}:=\left[f_{1}, f_{2}\right], g_{2}:=\left[f_{2}, g_{2}\right]$, on peut supposer de plus que $q_{2}>p_{2}$. On a alors

$$
\begin{aligned}
& X_{f_{1}}=\left(x^{p_{1}+1}+o\left(x^{p_{1}+1}\right)\right) \frac{\partial}{\partial x}, \quad X_{g_{1}}=\left(\alpha x^{q_{1}+1}+o\left(x^{q_{1}+1}\right)\right) \frac{\partial}{\partial x}, \\
& X_{f_{2}}=\left(\beta x^{p_{2}+1}+o\left(x^{p_{2}+1}\right)\right) \frac{\partial}{\partial x}, \quad X_{g_{2}}=\left(\gamma x^{q_{2}+1}+o\left(x^{q_{2}+1}\right)\right) \frac{\partial}{\partial x}
\end{aligned}
$$

avec $\alpha<0, \beta>0, \gamma<0$. D'autre part, il suffit clairement de se restreindre au demi-axe positif $\mathbb{R}^{+}$. Les dynamiques de $f_{1}$ et $g_{1}$ sont respectivement dilatantes et contractantes sur un intervalle de la forme $I=] 0, A]$ où $A$ est un réel positif suffisamment petit.

Posons $p:=p_{1}, q:=q_{1}$. Soit $\varphi \in \operatorname{Diff}(\mathbb{R}, 0)$ tangent à l'identité tel que $\varphi_{*} X_{f_{1}}=X_{p, \lambda}$. Réintroduisons la fonction $\psi_{p, \lambda}(x)=-\frac{1}{x^{p}}+\lambda \ln x$. Dans ce contexte, $\psi_{p, \lambda}$ définit un difféomorphisme $\mathcal{C}^{\infty}$ de $\varphi(I)$ sur un intervalle de la forme $J=]-\infty, \alpha], \alpha<0$ et $\psi_{p, \lambda_{*}} X_{p, \lambda}=\frac{\partial}{\partial w}$. Posons $\phi(x)=|x|^{\frac{-1}{p}}$; on observe alors que $\phi \circ \psi_{p, \lambda}(x)=x+o\left(x^{1+\varepsilon}\right)$ pour un certain $\varepsilon$ positif. Par conséquent, $\psi_{p, \lambda}{ }^{-1} \circ \phi^{-1}$ est de la même forme et ceci permet d'écrire $\psi_{p, \lambda}{ }^{-1}(x)=|x|^{\frac{-1}{p}}+O\left(x^{-\frac{1+\varepsilon}{p}}\right)$. Par un calcul élémentaire, on établit alors que $G_{1}(w):=\left(\psi_{p, \lambda} \circ \varphi\right) \circ g_{1} \circ\left(\psi_{p, \lambda} \circ \varphi\right)^{-1}(w)=w+a p|w|^{1-\frac{q}{p}}+$ $O\left(|w|^{1-\frac{q}{p}-\varepsilon}\right)$. Il est commode d'évaluer $G_{1}$ dans la coordonnée $\tilde{w}=$ $\eta(w)=-\frac{1}{q a}|w|^{\frac{q}{p}}$. On a alors

$$
\tilde{g}_{1}(\tilde{w}):=\eta \circ G_{1} \circ \eta^{-1}(\tilde{w})=\tilde{w}+1+O\left(\tilde{w}^{-\varepsilon}\right) .
$$

Lemme 19. Pour tout $N>0$,

$$
\tilde{g}_{1}^{N}(\tilde{w})=\tilde{w}+N+O\left(\frac{N}{\tilde{w}^{\varepsilon}}\right) .
$$

Preuve: On sait qu'il existe $C>0$ tel que pour tout $\tilde{w},|\tilde{g}(\tilde{w})-\tilde{w}-1| \leq$ $C \tilde{w}^{-\varepsilon}$. Par récurrence, on a

$$
\left|\tilde{g}_{1}^{N}(\tilde{w})-\tilde{w}-N\right| \leq C \sum_{n=0}^{N-1}\left|\tilde{g}_{1}^{n}(\tilde{w})\right|^{-\varepsilon} .
$$

On conclut par le fait que $\tilde{g}_{1}(\tilde{w})>\tilde{w}$.

Proposition 20. Soit $K$ un compact de $I=] 0, A]$. Alors, la suite

$$
F_{n}(t, w):=f_{1}^{n} \circ g_{1}^{\left[t n^{\frac{q}{p}-1}\right]} \circ f_{1}^{-n}
$$

converge uniformément sur $[0, T] \times K$ vers $\exp \left(t \delta X_{f_{1}}\right), \delta=a p^{1-\frac{p}{q}}$. 
Preuve: Les calculs qui suivent reproduisent à peu de choses près ceux menés dans $[\mathbf{L o}]$.

En revenant à la coordonnée initiale, on en déduit que

$$
G_{1}^{N}(w)=-|w|\left(1-N a q p^{\frac{-p}{q}}|w|^{\frac{-q}{p}}+|w|^{\frac{-q}{p}} O\left(\frac{N}{|w|^{\varepsilon}}\right)\right)^{\frac{p}{q}} .
$$

Fixons nous $T>0$ et soit $\mathrm{n}$ un entier positif. On veut évaluer

$G_{1}{ }^{N}(w-n)=-|w-n|\left(1-N a q p^{\frac{-p}{q}}|w-n|^{\frac{-q}{p}}+|w-n|^{\frac{-q}{p}} O\left(\frac{N}{|w-n|^{\varepsilon}}\right)\right)^{\frac{p}{q}}$

pour $N=\left[t^{\frac{q}{p}-1}\right], 0 \leq t \leq T$ (on rappelle que [ ] désigne la partie entière). On a $N=O\left(n^{\frac{q}{p}-1}\right)$ et par suite

$$
G_{1}^{N}(w-n)=-|w-n|\left(1-N a q p^{\frac{-p}{q}}|w-n|^{\frac{-q}{p}}+O\left(\frac{1}{n^{1+\varepsilon}}\right)\right)^{\frac{p}{q}} .
$$

Puisque $N=t n^{\frac{q}{p}-1}+O(1)$, on a

$$
G_{1}{ }^{N}(w-n)=-|w-n|\left(1-\frac{t}{n} a p^{\left(1-\frac{p}{q}\right)} \frac{|w-n|^{\frac{-q}{p}}}{n}+O\left(\frac{1}{n^{1+\varepsilon}}\right)\right) .
$$

Sur un compact $K$ de ] $-\infty, \alpha$ ], on hérite alors de l'estimation

$$
G_{1}^{N}(w-n)=w-n+\operatorname{tap}^{\left(1-\frac{p}{q}\right)}+O\left(\frac{1}{n^{\varepsilon}}\right) .
$$

Sachant maintenant que

$$
\left(\psi_{p, \lambda} \circ \varphi\right) \circ f_{1}^{n} \circ g_{1}^{\left[t n^{\frac{q}{p}-1}\right]} \circ f_{1}^{-n} \circ\left(\psi_{p, \lambda} \circ \varphi\right)^{-1}=G_{1}{ }^{\left[\operatorname{tn}^{\frac{q}{p}-1}\right]}(w-n)+n,
$$

la Proposition 20 est établie.

On dispose bien entendu du même résultat en substituant $f_{2}$ à $f_{1}$ et $g_{2}$ à $g_{1}$. Il existe alors un réel positif $\nu$ tel que pour $x>0$ assez petit, on ait

$$
\forall t \geq 0, \quad h \circ \exp \left(t X_{f_{1}}\right)(x)=\exp \left(t \nu X_{f_{2}}\right) \circ h(x) .
$$

A cette étape, on a donc établi que $h$ est $\mathcal{C}^{\infty}$ pour $x>0$. En substituant $g_{i}$ à $f_{i}$ et le commutateur $\left[f_{i}, g_{i}\right]$ à $g_{i}(i=1,2)$, on vérifie de même que $h_{*} X_{g_{1}}=\mu X_{g_{2}}$ avec $\mu>0$. Quitte à conjuguer $f_{1}, g_{1}$ par $x \rightarrow x^{\frac{1}{p}}$ où $p=\frac{p p c m\left(p_{1}, p_{2}\right)}{p_{1}}$ et $f_{2}, g_{2}$ par $x \rightarrow x^{\frac{1}{q}}$ avec $q=\frac{p p c m\left(p_{1}, p_{2}\right)}{p_{2}}$, on peut supposer que $p_{1}=p_{2}$. Les deux relations de conjugaison $h_{*} X_{f_{1}}=\nu X_{f_{2}}$, $h_{*} X_{g_{1}}=\mu X_{g_{2}}$ produisent, par élimination de $h^{\prime}$, une égalité du type

$$
h(x)=l \circ(k(x))^{\frac{p_{1}-q_{1}}{p_{1}-q_{2}}}
$$


avec $l, k \in \operatorname{Diff}(\mathbb{R}, 0), l^{\prime}(0), k^{\prime}(0)>0$. En réutilisant que $h_{*} X_{f_{1}}=\nu X_{f_{2}}$, on constate que nécessairement $q_{2}=q_{1}$, ce qui signifie que $h \in \operatorname{Diff}(\mathbb{R}, 0)$.

\section{Preuve du Théorème 3}

Soient $f, g \in \operatorname{Diff}(\mathbb{R}, 0)$ définis par

$$
\begin{aligned}
& f(x)=\frac{x}{1-x}, \\
& g(x)=\frac{x}{\left(1-x^{3}\right)^{\frac{1}{3}}} .
\end{aligned}
$$

S. White $[\mathbf{W h}]$ a montré que le groupe engendré par $f$ et $g$ est libre. Soient maintenant $F_{1}, G_{1}$ des difféomorphismes de l'intervalle $I=[-1,1]$ dans lui-même s'écrivant sous la forme:

$$
\begin{aligned}
& F_{1}(x)=x+\psi_{1}(x), \\
& G_{1}(x)=x+\psi_{2}(x),
\end{aligned}
$$

où les $\psi_{i}, i=1,2$ sont

- De classe $\mathcal{C}^{\infty}$ sur $I$.

- Nuls exactement en $-1,0$ et -1 .

- Plats en -1 et 1 et strictement supérieurs à -1 sur $I$.

Enfin, on peut les choisir de telle sorte que $F_{1}$ et $G_{1}$ coïncident respectivement avec $f$ et $g$ au voisinage de l'origine. Considérons les deux familles de difféomorphimes de $I$ :

$$
\begin{gathered}
F_{\varepsilon}(x)=x+\varepsilon \psi_{1}(x), \\
G_{\varepsilon}(x)=x+\varepsilon \psi_{2}(x),
\end{gathered}
$$

où $\varepsilon$ est un paramètre décrivant l'intervalle $\mathbb{R}$.

Pour tout $\varepsilon$, on notera $\mathcal{G}_{\varepsilon}$ le sous groupe de $\operatorname{Diff}(\mathbb{R}, 0)$ engendré par $F_{\varepsilon}$ et $G_{\varepsilon}$ (vus comme germes à l'origine). Remarquons que les éléments de $\mathcal{G}_{\varepsilon}$ sont analytiques. De plus, les familles $\left(F_{\varepsilon}\right)$ et $\left(G_{\varepsilon}\right)$ dépendent analytiquement du paramètre $\varepsilon$; pour tout $n \in \mathbb{Z}$, il en résulte que les familles des itérés d'ordre $n\left(F_{\varepsilon}{ }^{n}\right)$ et $\left(G_{\varepsilon}{ }^{n}\right)$ dépendent également analytiquement de $\varepsilon$. Fixons $m \in \mathbb{N}^{*}$ et soit $\mathcal{M}_{m}^{\varepsilon}$ l'ensemble des mots de longueur $m$ en $F_{\varepsilon}, G_{\varepsilon}$. Par analyticité et sachant que $\mathcal{G}_{1}$ est libre, on déduit que $\mathcal{M}_{m}^{\varepsilon}$ contient l'identité seulement pour un ensemble de points isolés de $\mathbb{R}$.

Maintenant, soit $\Lambda=\left\{\varepsilon \in \mathbb{R}\right.$ tel que $\mathcal{G}_{\varepsilon}$ soit un groupe libre à deux générateurs\}. Le théorème de Baire nous assure que $\Lambda$ est dense dans $\mathbb{R}$. 
Définition. Soit $\mathcal{C} \subset \mathbb{R}$ un ensemble de Cantor. On dira que $\mathcal{C}$ est homogène si

- Chaque composante connexe de $\mathbb{R} \backslash \mathcal{C}$ est bornée.

- Pour tout intervalle compact $J$ dont l'intérieur est d'intersection non vide avec $\mathcal{C}$, la dimension de Haussdorf de $J \cap \mathcal{C}$ est indépendante de $J$.

Soient $\mathcal{C}_{1}, \mathcal{C}_{2}$ deux ensembles de Cantor homogènes de $\mathbb{R}$ de dimension de Haussdorf respectives $d_{1}<d_{2}$. Donnons nous une énumération $\left(I_{n}\right)_{n \in \mathbb{N}}$ des composantes connexes de $\mathbb{R} \backslash \mathcal{C}_{1}$. On peut supposer qu'on a choisi $\mathcal{C}_{1}$ et $\mathcal{C}_{2}$ de telle sorte que:

a) $0 \in \mathcal{C}_{1} \backslash \bigcup_{n \in \mathbb{N}} \overline{I_{n}}$.

b) $\varphi\left(\mathcal{C}_{1}\right)=\mathcal{C}_{2}$ et $\varphi(0)=0$ où $\varphi$ est un homéomorphisme de $\mathbb{R}$ préservant l'orientation et affine en restriction à chaque composante $I_{n}$.

Pour tout $n \in \mathbb{N}$, on posera $J_{n}=\varphi\left(I_{n}\right)$. Soit $\psi_{n}$ et $\xi_{n}$ les transformations affines conservant l'orientation envoyant respectivement $[-1,1]$ sur $I_{n}$ et $J_{n}$. Choisissons maintenant une suite $\left(\varepsilon_{n}\right)$ d'éléments de $\Lambda$ convergeant vers 0 de telle sorte que pour tout $n>1$ et pour tout $1<i \leq n$ :

$$
\begin{aligned}
& \sup _{x \in I_{n}}\left|\left(\psi_{n} \circ F_{\varepsilon_{n}} \circ \psi_{n}^{-1}\right)^{(i)}(x)\right| \leq \frac{1}{n}, \\
& \sup _{x \in I_{n}}\left|\left(\psi_{n} \circ G_{\varepsilon_{n}} \circ \psi_{n}^{-1}\right)^{(i)}(x)\right| \leq \frac{1}{n}, \\
& \sup _{x \in J_{n}}\left|\left(\xi_{n} \circ F_{\varepsilon_{n}} \circ \xi_{n}{ }^{-1}\right)^{(i)}(x)\right| \leq \frac{1}{n}, \\
& \sup _{x \in J_{n}}\left|\left(\xi_{n} \circ G_{\varepsilon_{n}} \circ \xi_{n}{ }^{-1}\right)^{(i)}(x)\right| \leq \frac{1}{n} .
\end{aligned}
$$

La collection $\left(\psi_{n} \circ F_{\varepsilon_{n}} \circ \psi_{n}{ }^{-1}\right)_{n \in \mathbb{N}}$ définit une bijection de l'ensemble $\bigcup_{n \in \mathbb{N}} \bar{I}_{n}$ sur lui même qui s'étend en un difféomorphisme de classe $\mathcal{C}^{\infty}$ de $\mathbb{R}$ valant l'identité en restriction à $\mathcal{C}_{1}$. On notera $F$ le germe de $\operatorname{Diff}(\mathbb{R}, 0)$ induit par ce difféomorphisme. De même, les collections $\left(\psi_{n} \circ\right.$ $\left.G_{\varepsilon_{n}} \circ \psi_{n}{ }^{-1}\right),\left(\xi_{n} \circ F_{\varepsilon_{n}} \circ \xi_{n}{ }^{-1}\right)$ et $\left(\xi_{n} \circ G_{\varepsilon_{n}} \circ \xi_{n}{ }^{-1}\right)$ induisent à l'origine de $\mathbb{R}$ des germes notés respectivement $G, \tilde{F}$ et $\tilde{G}$.

Par construction, les groupes $\langle F, G\rangle$ et $\langle\tilde{F}, \tilde{G}\rangle$ sont topologiquement conjugués. A cause de l'invariance de la dimension de Haussdorf en classe $\mathcal{C}^{\infty}$, on constate qu'ils satisfont l'énoncé du Théorème 3 . 


\section{Bibliographie}

[Ce-Mo] D. Cerveau et R. Moussu, Groupes d'automorphismes de $(\mathbf{C}, 0)$ et équations différentielles $y d y+\cdots=0$, Bull. Soc. Math. France 116(4) (1988), 459-488 (1989).

[E] J. ÉCALLE, "Les fonctions résurgentes", Tome I. Les algèbres de fonctions résurgentes, Publications Mathématiques d'Orsay 81-5, Université de Paris-Sud, Département de Mathématique, Orsay, 1981; Tome II. Les fonctions résurgentes appliquées à l'itération, Publications Mathématiques d'Orsay 81-6, Université de Paris-Sud, Département de Mathématique, Orsay, 1981, pp. 248-531; Tome III. L'équation du pont et la classification analytique des objects locaux, Publications Mathématiques d'Orsay 85-5, Université de Paris-Sud, Département de Mathématiques, Orsay, 1985.

[K] T. KimurA, On the iteration of analytic functions, Funkcial. Ekvac. 14 (1971), 197-238.

[Lo] F. LORAY, 5 leçons sur la structure transverse d'une singularité de feuilletage holomorphe en dimension 2 complexe, leçons 1 et 2, Monographies du réseau Red TMR Europea, Sing. Ec. Dif. Fol. 1 (1998).

[Ma] B. Malgrange, Travaux d'Écalle et de Martinet-Ramis sur les systèmes dynamiques, in: "Bourbaki Seminar, Vol. 1981/1982", Astérisque 92-93, Soc. Math. France, Paris, 1982, pp. 59-73.

[Na] I. NAKAI, Separatrices for nonsolvable dynamics on C, 0, Ann. Inst. Fourier (Grenoble) 44(2) (1994), 569-599.

[Ra] J.-P. RAmis, Théorèmes d'indices Gevrey pour les équations différentielles ordinaires, Mem. Amer. Math. Soc. 48(296) (1984), 95 pp.

[Sh] A. A. Shcherbakov, Density of the orbit of a pseudogroup of conformal mappings and generalization of the Khudaü-Verenov theorem, (Russian), Vestnik Moskov. Univ. Ser. I Mat. Mekh. 4 (1982), 10-15, 84 .

[Ta] F. TAKEns, Normal forms for certain singularities of vectorfields, Colloque International sur l'Analyse et la Topologie Différentielle (Colloques Internationaux du Centre National de la Recherche Scientifique, Strasbourg, 1972), Ann. Inst. Fourier (Grenoble) 23(2) (1973), 163-195. 
[To] J.-C. Tougeron, An introduction to the theory of Gevrey expansions and to the Borel-Laplace transform with some applications, Preprint Univ. Toronto, Canada (1990).

[Wh] S. White, The group generated by $x \mapsto x+1$ and $x \mapsto x^{p}$ is free, J. Algebra 118(2) (1988), 408-422.

I.R.M.A.R. Campus de Beaulieu

Université de Rennes 1

Rue du Général Leclerc

35042 Rennes

France

E-mail address: ftouzet@maths.univ-rennes1.fr

Primera versió rebuda el 4 d'octubre de 2002, darrera versió rebuda el 19 de maig de 2003. 